\title{
A Novel Load Forecasting Model for Automatic Fault Clearance in Secondary Distribution Electric Power Grid using an Extended-Multivariate Nonlinear Regression
}

\author{
Hussein Bakiri*, Hellen Maziku*, Nerey Mvungi*, Ndyetabura Hamisi*, Massawe Libe** \\ *Department of Computer Systems Engineering, College of Information and Communication Technologies, a researcher at the \\ department, P.O.Box 33335, Dar es Salaam, Tanzania \\ **Department of Electronics and Telecommunication Engineering, College of Information and Communication Technologies, \\ researcher at the department, P.O.Box 33335, Dar es Salaam, Tanzania
}

(hussein.bakiri@ifm.ac.tz, maziku.hellen@udsm.ac.tz,nhmvungi@udsm.ac.tz,yhamisi@udsm.ac.tz, massawe.libe@udsm.ac.tz)

*Corresponding Author; Hussein Bakiri, Postal address, Tel.: +255 718572 299; E-mail address: hussein.bakiri@ifm.ac.tz

Received: 24.04.2021 Accepted:17.06.2021

\begin{abstract}
Smart grid is an emerging platform adopted by many electric power utility companies to enhance proper service delivery as well as cost-effective operations. Automatic Fault Detection and Clearance (AFDC) is a part of intelligent technology initiatives established on Tanzania's grid aiming at detecting, managing, and handling fault with little or without human intervention. Being one of the components of AFDC, the Load Forecasting (LF) plays important role in feeding restoration and distributed energy resource agencies. However, the efficiency of the existing LF approaches is found to be compromised when it comes to data whose distribution is characterized by a random-walk behavior. Therefore, this research work proposes an efficient AFDC-based LF (LF-AFDC) which is capable of generating load demand profile based on fault data. Firstly, the design requirements of the LF-AFDC framework are established using focus group discussion and literature survey. Secondly, the design of the LF model is achieved through parameter calibration from the existing Multivariate Non-Linear Regression (MNLR) method. Thirdly, the design of the LF-AFDC framework is achieved based on the design requirements for the realtime and fault-driven forecasting. Findings indicate the capability of the proposed framework to forecast the next load profile from the given fault-date, fault-time, restoration duration, Gross-Domestic Product (GDP), number of customers, and population information. Furthermore, the simulation results indicate the capability of the extended MNLR (e-MNLR) method to outperform the ANN, SVM, KNN, MICE, MissForest and MNLR models.
\end{abstract}

Keywords - Smart Grid, Automatic Fault Clearance, Load Forecasting, Extended-MNLR

\section{Introduction}

Smart grid is regarded as a modern electric power grid infrastructure adopted by utility companies to enhance service efficiency, availability, and reliability [1]. In Tanzania, for instance, the primary distribution part is largely automated but little attention has been paid to the secondary distribution. Thus, in the effort to establish smart distribution technologies in the Secondary Distribution Electric Power Grid (SDEPG), the University of Dar es Salaam in conjunction with the Swedish International Development Agency (Sida), has facilitated research focusing on establishing an AFDC [2], [3].

An AFDC is a proposed smart grid mechanism aiming at detecting and managing faults in the SDEPG, with little or no physical interaction at all [2]. The AFDC mechanism works in 
the sense that, when a fault occurs in the SDEPG, it is detected by a fault management agent and later on its localization is identified [4]. Following the fault identification process, the healthy part is isolated and then the service is restored by the restoration controller agency using Distributed Energy Resources (DER) [4]. However, restoration controllers and DER have no built-in mechanisms for anticipating the power consumption demand for a particular distribution area. Therefore, there is a need for adapting existing load forecasting models to suit the AFDC requirements.

Several research works such as [4]-[12], have attempted to use load forecasting for service restoration. Their proposed mechanisms have limitations when it comes to forecasting in a faulty-based environment as well as when data is characterized by random-walk distribution. In the fault-based occasion environment, the generation of a load profile is supposed to be driven by information associated with the fault such as time, date, and restoration span. This research work, therefore, proposes a fault and restoration-based forecasting mechanism that generates load profiles for both DERs and restoration controller agencies. The core part of the proposed forecasting mechanism is built on top of the extended-MNLR that works better in the situation of random-walk data.

\section{Background}

\subsection{Automatic Fault Clearance}

AFDC refers to the practice that encompasses mechanisms for detecting, handling, and managing faults at Secondary Distribution Electric Power Grid (SDEPG) with little or without human intervention[2], [3], [13]. The diagram in Fig.1 illustrates the overall component interaction of the AFDC at the SDEPG. The network is supposed to be comprised of three subnetworks served by transformers connected to the main distribution line.

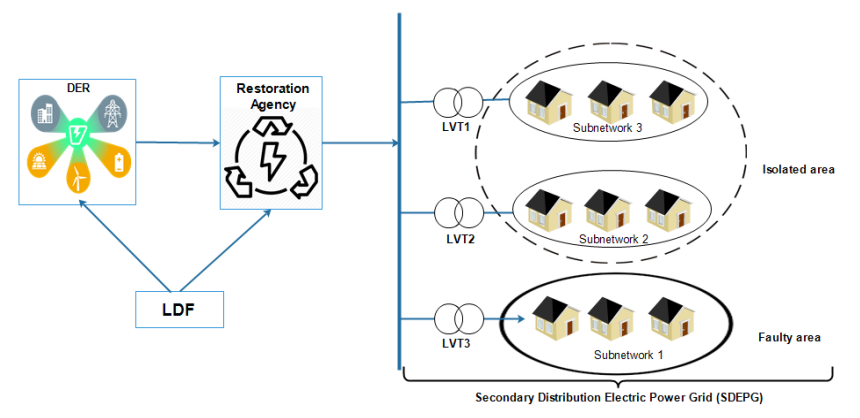

Fig. 1. The role of LF mechanism in the overall AFDC context

The AFDC mechanism is expected to work in such a way that, when the fault occurs; let's say in subnetwork 1, it is detected by the fault agency. The faulty subnetwork is automatically isolated and Service Restoration (SR) agency resumes power to the rest of the unaffected parts (subnetwork2 and 3) using available DERs. Thus, the role of the LF mechanism in the AFDC is to anticipate the real-time power supplied to the isolated health part during the restoration process.

\subsection{Load Forecasting for Service Restoration}

A method for estimating the time at which electric power will be restored is proposed by [7] using the company's experience in six hurricanes, eight storms, and accelerated failure time. In the effort to adapt forecasting models for automated service restoration management. [6] have proposed an algorithm that uses day type and day time to determine a pre-fault load profile for online estimation of loads in a network. The pre-fault short-term load profile is then used to feed the capacity check algorithm of the power restoration controller. [10] proposes a forecasting mechanism that generates a short-term load profile which is then used to feed an automatic frequency restoration reserve from renewable energy based on a virtual power plan. [14] Proposes a flexible model for predicting electric power required to be restored by distributed generation controls in an uncertain environment. [11] proposes a short-term load forecasting approach that can be used to model load demand forecasting for 20-minutes service restoration in secondary distribution power networks in Tanzania.

All aforementioned approaches for service restoration are limited to forecasting in three aspects; firstly, in the faultdriven situation in which the load profile is automatically generated by the trigger from the fault management agency. Secondly, in the situation where the forecasting is also based on the information from a service restoration agency. Thirdly, in the situation where load data characterized by random-walk distribution.

\subsection{Load forecasting methods}

Load forecasting methods range from statistical, regression to machine learning [15]. Statistical-based forecasting methods include Box-Jenkins, Calman filtering algorithm, grey model, Automatic regression, and exponential smoothing [15]. Regression-based forecasting methods include Autoregressive Moving Average (ARMA), Autoregressive Integrated Moving Average (ARIMA), Autoregressive Moving Average (ARMA), and Autoregressive Moving Average with exogenous input (ARMAX) [16]. Statistical and regression methods have a limited ability to model nonlinear and nonstationary distribution. Thus, to accommodate nonlinear behavior in regression models, [17] has proposed a new method that can forecast in the nonlinear fashion.

The extension of statistical and regression methods to accommodate nonlinear behavior does not efficiently address the nonstationarity and nonlinearity bottlenecks. Contrary, machine learning models seem to work better with nonlinear data and they are well fitted for short-term forecasting relative to regression models [16]. However, the capability of the machine learning models such as ANN, SVM, LSTM, and 
fuzzy logic might be compromised when it comes to the load data that exhibits random-walk behavior.

\subsection{Factors to be Considered when Designing Load Forecasting Model in Developing Countries}

Research works have reported on the existence of peculiar long-term load distribution in developing countries contrary to the developed ones. The peculiarity is caused by socialeconomic and technological grounds [18]-[21]. The exhibited abnormal load pattern in developing countries, which is characterized by non-stationarity, hinders the efficiency of existing forecasting methods [22]. On the other hand, the daytime, calendar events, GDP, number of customers, electricity price, and population have been reported to influence long-term electricity consumption [21]-[27]. Therefore, it is necessary to investigate specific determinants of power consumption in a particular country.

\subsection{The MNLR model}

The MNLR model has been proposed in the work by [17]. The model has produced promising results when applied to nonlinear time series data. Moreover, the model can simply be extended through parameterization. The original form of the MNLR model is shown in equation (1).

$$
\mathrm{Y}=\alpha_{0}\left(x_{1}^{\alpha_{1}}\right)\left(x_{2}^{\alpha_{2}}\right) \ldots x_{n}^{\alpha_{n}}
$$

Where $\alpha_{0}$ to $\alpha_{n}$ model parameters, $x_{1}$ to $x_{n}$ are input variables and $\mathrm{Y}$ is a dependent variable.

\section{Material and Methods}

\subsection{Research Design}

The training and testing data in this study are based on secondary records. Data collection is achieved qualitatively whereas the data analysis is conducted quantitatively. After the analysis, the data is then tested on six widely used prediction methods namely; MNLR, MissForest, SVM, ANN, $\mathrm{KNN}$, and MICE to test the claim that existing methods do not work effectively on random-walk distribution. Later on, the method presented by equation (1) is extended so that it is capable of forecasting effectively in random-walk data. Finally, the proposed LF model is designed according to the requirements of the AFDC mechanism. The overall research methodology is presented in Fig. 2.

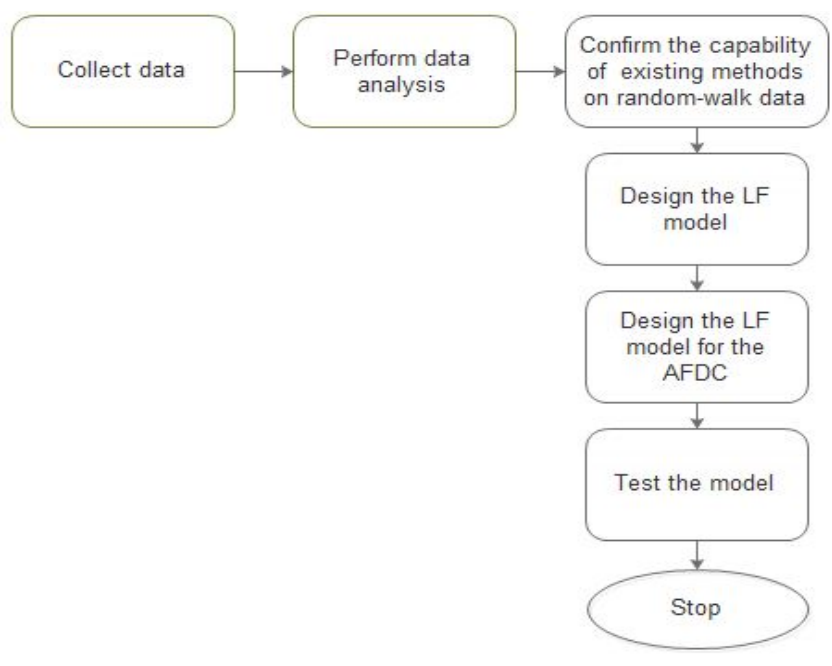

Fig. 2. The overall research design process

\subsection{Study Area and Data Description}

This study is based on the Mikocheni area; a secondary distribution network having three transformers named BBQ Village SS1, Abiudi Street, and Kimweri Avenue-ss1 (with the power of $315 \mathrm{KVA}, 200 \mathrm{KVA}$, and 100KVA respectively), serving an average of 143 customers per transformer. Each transformer has an average consumption of $30 \mathrm{KWh}$ per 20 minutes.

The study area is depicted by a site map in Fig. 3. The area is characterized by customers of moderate GDP earning, primarily residential buildings. Furthermore, the area is characterized by annual consumption growth as per 2015 to 2018 data as presented in Fig. 4.

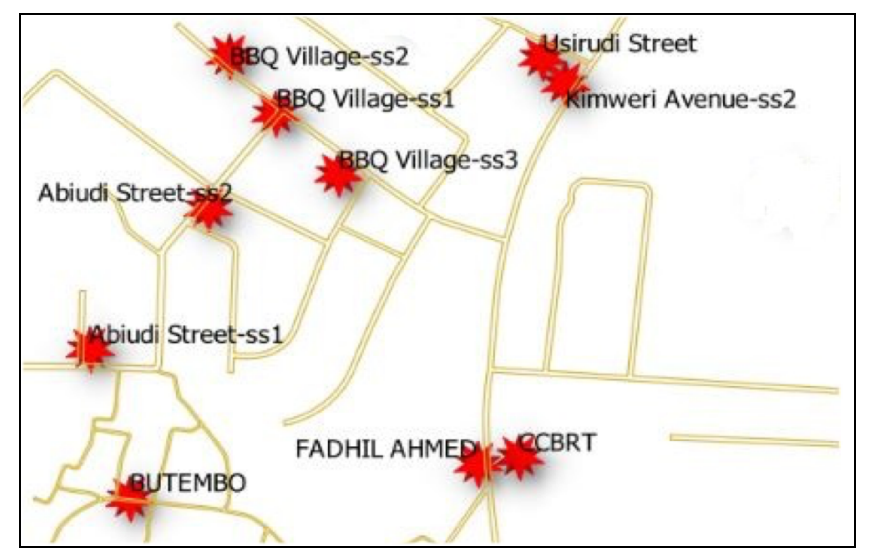

Fig. 3. A site map of the secondary distribution network at Kinondoni-North (Mikocheni) study area in Dar es salaam 


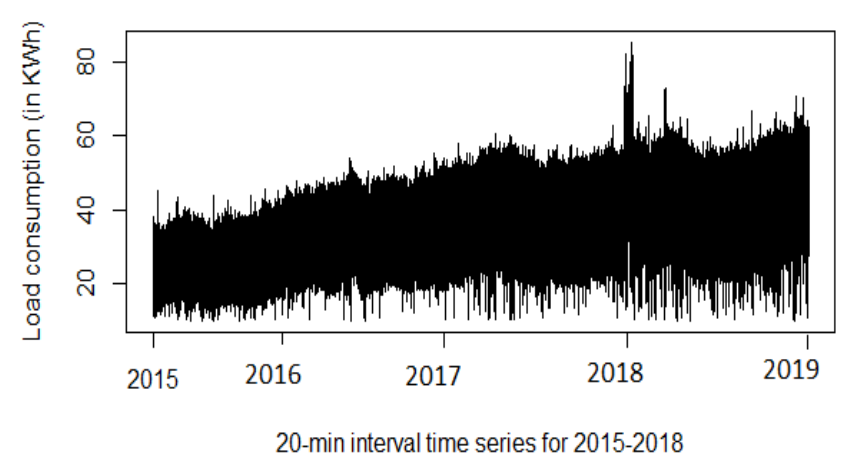

Fig. 4. The daily load consumption from 2015-2018 as per Kinondoni-North data in Dar es Salaam

\subsection{Data collection}

The 20-minute interval load data from 2015 to 2019 (a total of 105193 records) has been recorded through accumulated meter reading situated on the study area. Moreover, the annual GDP data from 2015 to 2019 was collected from the National Bureau of Statistics (NBS). Furthermore, the monthly number of customers and population data (from 2015 to 2019) was also collected from Tanzania National Electric Supply Company (TANESCO).

\subsection{Data Analysis}

The collected load data is checked for the presence of outliers and missing values using the $\mathrm{k}$-mean algorithm. The k-mean is a clustering and supervised machine learning algorithm that works based on the specified number of clusters and the central mean values of each cluster (centroids) [28]. The choice of the k-mean algorithm in this work is based on the fact that the central values are already known from expert knowledge. Therefore, two clusters are formed for which two centroid values (4 and 25) are initialized. The setting of centroid values is based on the expert knowledge that the average power consumption is 16 , and thus any values below 15 are regarded as outliers.

\subsection{Determining Characteristics of Load Distribution in Tanzania}

Autocorrelation Function (ACF) and Partial Autocorrelation Function (PACF) are used to test for randomwalk behavior in the time series data. Furthermore, a load graph against time for the five years (2015 to 2019) is also plotted to aid in visual analysis.

\subsection{Checking for the Capability of Existing Forecasting Methods on the Random-Walk Data}

The six widely used methods (ANN, SVM, KNN, MNLR, MissForest, and MICE) are applied to the available randomwalk data to confirm their prediction capability. If poor prediction accuracy is observed, this will then lead us to extend an existing model to improve predictive accuracy. Furthermore, choosing which among the models to adapt is based on the easily adaptable method that can suit the nature of the data.

\subsection{The LF Model}

The LF model is designed such that it is capable of producing promising results despite the random-walk nature of the load data. In addition to that, the model is designed to achieve a high level of flexibility using both long (GDP, number customer, and population) and short-term (daytime, day type, and temperature) factors. Moreover, due to its ability to be extensible, the LF model is built on top of the MNLR model presented in equation (1). The overall design process of the LF model is presented in Fig. 5. Finally, the parameters and variables in equation (1) are calibrated to produce a new e-MNLR equation.

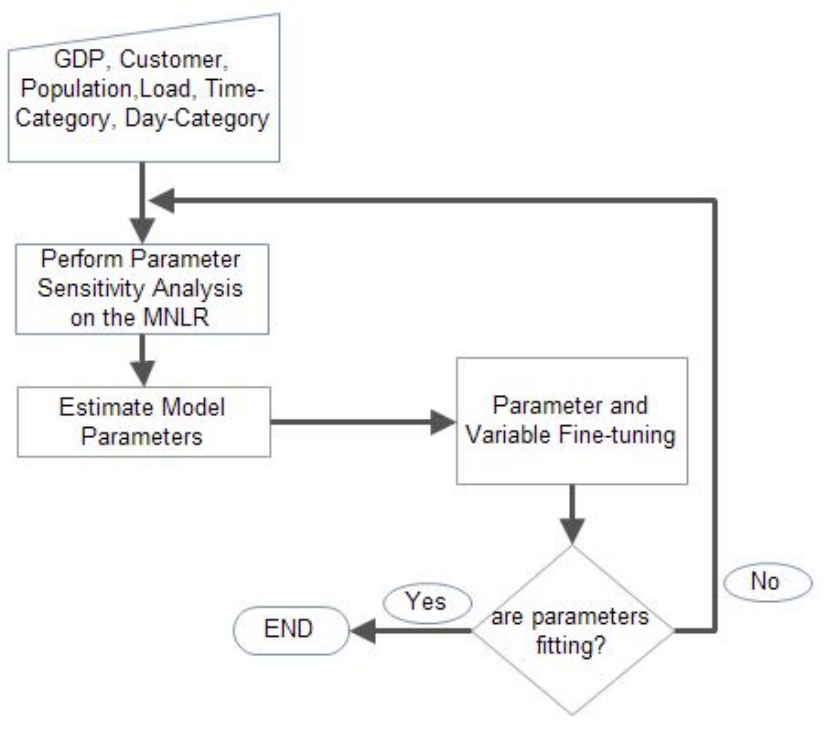

Fig. 5. The architecture of the proposed LF model

\section{Designing the LF Model}

\subsection{Conceptual Model of the Proposed LF-AFDC Mechanism}

To design an effective LF-AFDC framework, it is required to be modeled in a way that it is capable of generating a 20-minutes load profile once the fault is detected. Furthermore, the model should also be designed in a way that it receives fault date and fault time from the fault detection agency. In addition to that, the LF-AFDC mechanism should also receive restoration duration from a service restoration or maintenance agency.

The model should account for the GDP, the number of customers and population as these are the main determinants of long-term electricity consumption in Tanzania. The conceptual model of the proposed LF-AFDC framework is clearly illustrated in Fig. 6 . The overall modeling process is portrayed by the Algorithm I pseudocode in Table 1. 


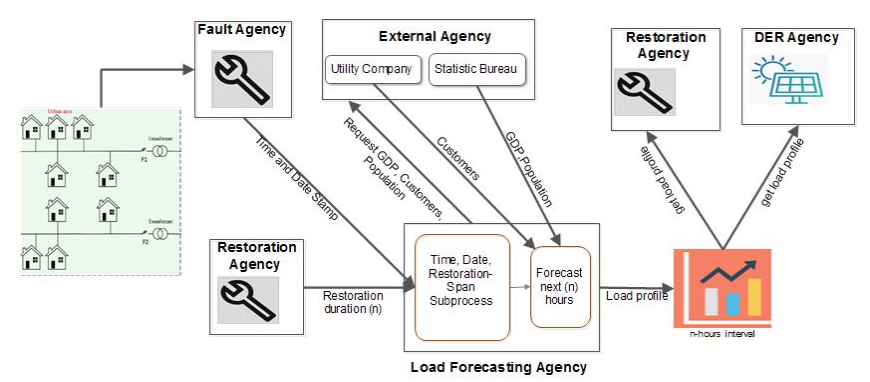

Fig. 6. The Conceptual model of the proposed LF-AFDC framework

The outputs of time, date, and restoration-transformation procedures become the corresponding time, day type, and 20minute interval values respectively. Time, date, and restoration duration together with values of GDP, the number of customers, and population become inputs to the e-MNLR model as seen in Fig. 7.
Table 1. The LF-AFDC algorithm

\begin{tabular}{|l}
\hline The algorithm I: LF-AFDC \\
\hline $\begin{array}{l}\text { 1: Receive real-time GDP,customers, population, fault time (ft), fault } \\
\text { date (fd) and restoration duration (rd) stamps }\end{array}$ \\
2: Compute forecasting span and number of forecasting intervals using \\
$\quad$ rd \\
3: Compute time and day ordinal values using time_transformation and \\
$\quad$ day_transformation sub_processes \\
4: Forecast load profile using the e-MNLR from equation (2) \\
5: Produce forecasted load profile at the time interval of length (rd) \\
6: End
\end{tabular}

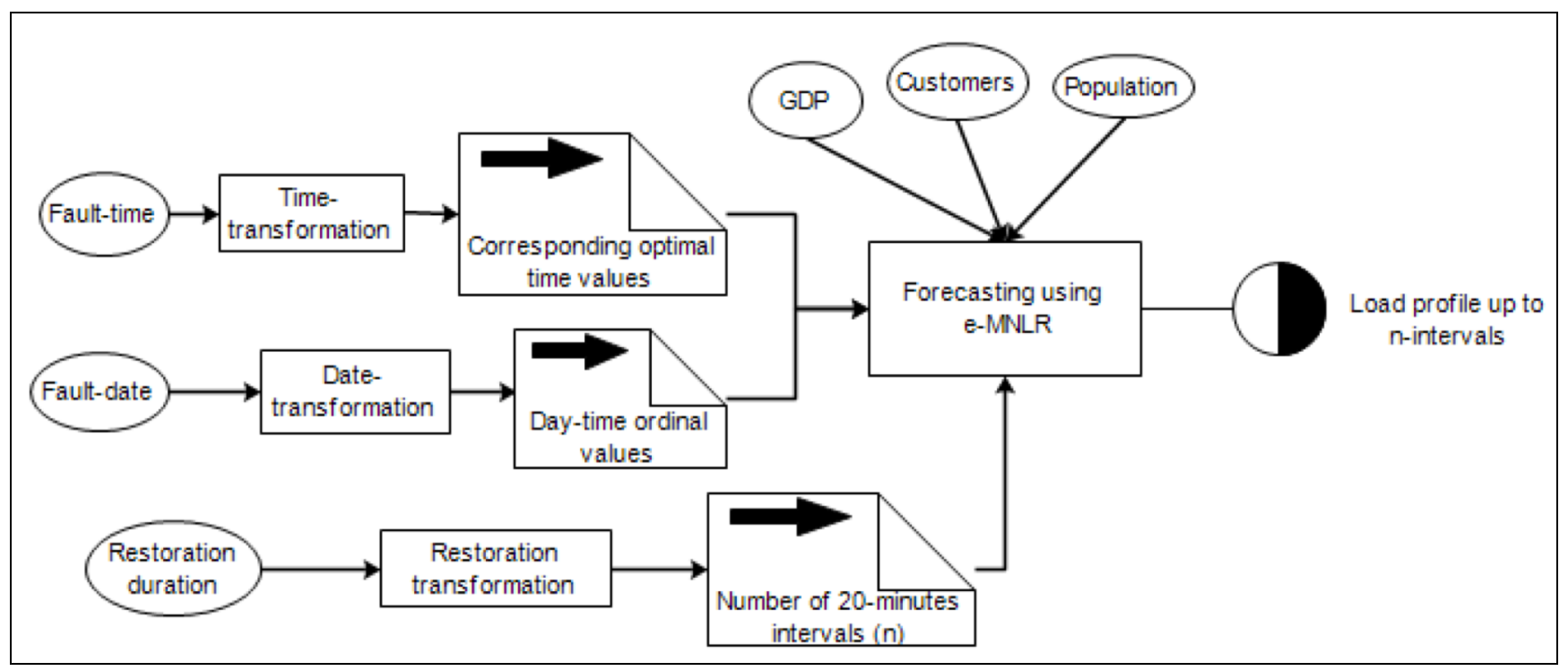

Fig. 7. The architectural model of the proposed LF in AFDC

Therefore, after parameterizing the original MNLR equation (from equation (1), with the design requirements in Fig. 6, the extended model becomes as shown in equation (2).

$$
y_{t}=x_{t}^{\alpha_{t}} * x_{d}^{\alpha_{d}} * x_{g}^{\alpha_{g}} * x_{p}^{\alpha_{p}} * x_{c}^{\alpha_{c}}
$$

Where $y_{t}=$ load at time (20 minutes), $x_{t}=$ time value whose load is sought, $x_{d}=$ day category number, $x_{g}=$ GDP value of the current year, $x_{p}=$ current population, $x_{c}=$ current number of customers., and $\alpha_{t}, \alpha_{d}, \alpha_{g}, \alpha_{p}$ and $\alpha_{c}$ are time, day, GDP, population and customer parameters respectively.

\subsection{Time Transformation Sub-process}

The corresponding time value $\left(x_{t}\right)$ in each time category is obtained from applying a logarithmic function to the equation (5). The parameter variable is expressed in equation (3).

$$
x_{t}=\log ^{-1}\left(\frac{\left(\left[\log \left(y_{t}\right)\right]-\left[\alpha_{d} * \log \left(x_{d}\right)+\alpha_{g} * \log \left(x_{g}\right)+\alpha_{p} * \log \left(x_{p}\right)+\alpha_{c} * \log \left(x_{c}\right)\right]\right)}{\alpha_{t}}\right)
$$

The received fault time stamp from the fault agency needs to be transformed into time categories $\left(y_{\text {cat }}\right)$. Firstly, the time is transformed into the corresponding numerical values $(x)$ so that it is in a computed form. Secondly, the obtained numerical values, are transformed into the equivalent time category. Thirdly, the stepwise function is generalized to produce equivalent time values for any 
inputted time. The formulated stepwise function is shown in equation (4).

$$
f\left(y_{\text {cat }}\right)=\left\{\begin{aligned}
x_{\text {mid }}-0.1, & x_{\text {cat }}=\text { midnight } \\
x_{\text {lmid }}-0.05, & x_{\text {cat }}=\text { late midnight } \\
x_{\text {dawn }}-0.05, & x_{\text {cat }}=\text { dawn } \\
x_{\text {morn }}-0.025, & x_{\text {cat }}=\text { morning } \\
x_{\text {lmorn }}+0.1, x_{\text {cat }} & =\text { late morning } \\
x_{\text {noon }}-0.05, & x_{\text {cat }}=\text { noon } \\
x_{\text {afnoon }}-0.05, & x_{\text {cat }}=\text { afternoon } \\
x_{\text {evening }}-0.5, & x_{\text {cat }}=\text { evening } \\
x_{\text {levening } 1}+0.15, & x_{\text {cat }}=\text { late evening } 1 \\
x_{\text {levening } 2}+0.15, & x_{\text {cat }}=\text { late evening } 2 \\
x_{\text {night }}-0.1, & x_{\text {cat }}=\text { night }
\end{aligned}\right.
$$

\subsection{Date Transformation Sub-process}

The received date information from the faulty detection agency needs to be processed to extract the day number. The day number is then used to determine the day category. Using the day category, the ordinal day values are deduced.

\subsection{Restoration Duration Sub-process}

It is important to determine the starting and ending forecasting times in advance, to produce the load profile that will be used throughout the restoration duration. Furthermore, the number of time values between starting ending points needs to be identified as well. To achieve this, the received fault time and restoration duration are converted into minutes (from hours) and then their summation is computed. The overall restoration process is depicted in Fig. 8.

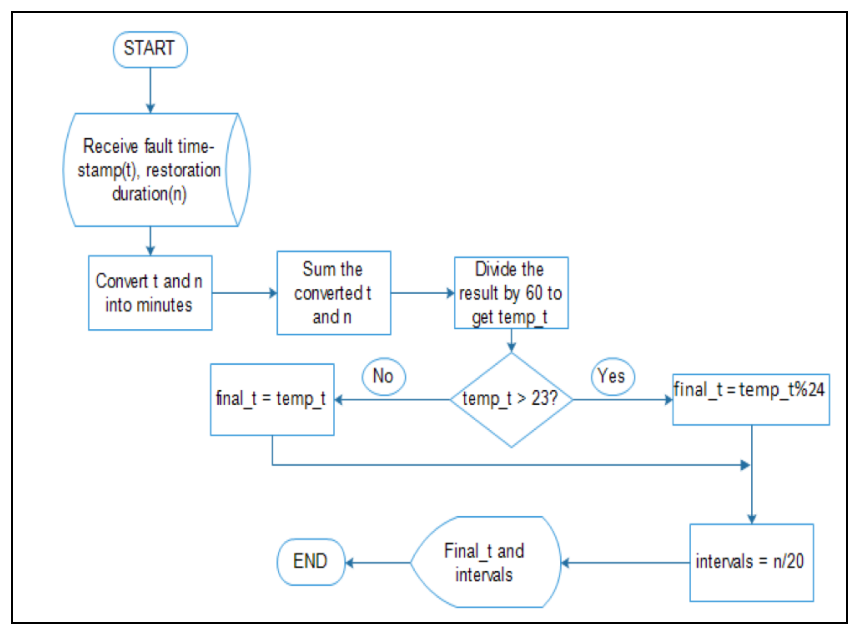

Fig. 8. Restoration-duration transformation process

\section{Simulation Results and Discussion}

5.1. Results

\subsubsection{Long-term Load Distribution Characteristics in Tanzania}

The test conducted on 2015 to 2018 data indicates that the load distribution trend in Tanzania is characterized by random-walk behavior. Firstly, from Fig. 9-a (autocorrelation versus lags), it can be seen that a gentle decline for the number of lags is observed to span towards the bottom margin of a significant correlation line. Secondly, from Fig. 9-b (partial autocorrelation versus lags) it is observed that the first lag shows a significant correlation while the rest are insignificant. Furthermore, Fig. 9-c confirms the nonstationary behavior exhibited by the time series data. Therefore, the aforementioned graph analysis confirms the random-walk property in the load data

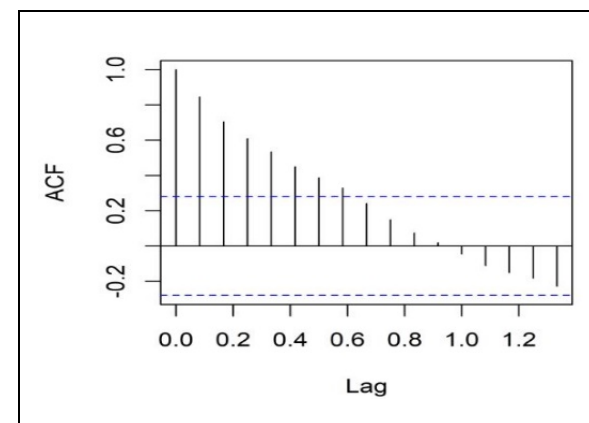

a

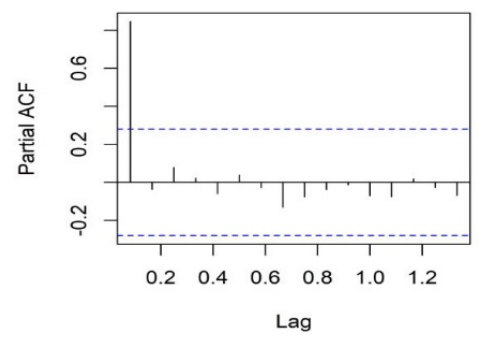

b

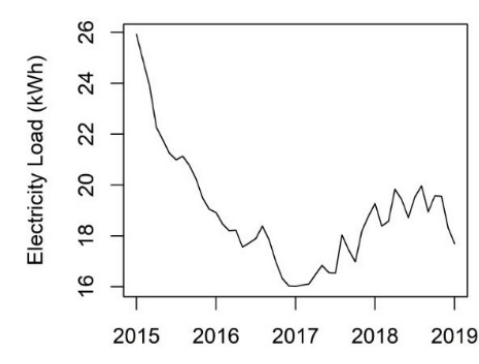

c

Fig. 9. Random-walk test results using 2015 to 2018 North-Kinondoni load data in Dar es Salaam

\subsubsection{Outliers and Missing Values in the Load Data}

After running the k-mean algorithm in $\mathrm{R}$, the empirical results indicate that the load data contains about 5.17852\% (which is 5207 out of 105192) outliers. This finding confirms the findings observed from other research works that load data in developing countries might be characterized by the presence of corrupt entries. These 
corrupt entries may jeopardize the accuracy of the prediction models if not treated beforehand.

\subsubsection{Are the Existing Methods Effective on a Random-Walk Data?}

The six widely used methods (ANN, SVM, MissForest, $\mathrm{KNN}$, MICE, and MNLR) are implemented in the R language. The simulation results presented in Table 2 show the RMSE, MAE, and MAPE values of the six predictive methods. As presented in the table, the result is not such promising and therefore these values reflect the incapability of the chosen methods on the time series data characterized by random-walk.

Table 2. Simulation results from the six most widely used predictive methods

\begin{tabular}{|c|c|c|c|c|}
\hline Method & Execution Environment & RMSE & MAE & MAPE \\
\hline MNLR & N/A & 12.77399 & 9.226775 & 34.34231 \\
\hline Miss-Forest & $\begin{array}{l}\text { 8GB RAM, Co-i5, Max. iterations }=\text { Default, Sample Size }=16055 \text {, } \\
\text { Training Data }=84154\end{array}$ & 3.035463 & 2.732547 & 10.24622 \\
\hline$S V M$ & $\begin{array}{l}\text { 8GB RAM, Co-i5, Kernel }=\text { radio, } \text { Type }=\text { eps-regression, } \\
\text { Training Data }=5000\end{array}$ & 5.32299 & 3.15148 & 11.35937 \\
\hline$A N N$ & $\begin{array}{l}\text { 8GB RAM, Co-i5, No. Hidden Layers }=2 \text {, Hidden Neurons }=4 \\
\text {,Threshold }=0.001 \text {, Algorithm }=\text { rprop }+ \text {, Activation Function }=\text { Logistic, } \\
\text { err.fct }=\text { sse, StartWeight }=1.3 \text {, StepMax }=20,000\end{array}$ & 6.9456 & 7.466061 & 27.8525 \\
\hline MICE & 8GB RAM, Co-i5, Max. iterations $=150, \mathrm{M}=5$, Method $=$ sample & 12.09854 & 9.361562 & 33.43858 \\
\hline$K N N$ & 8GB RAM, Co-i5, variable $=$ load, $\mathrm{k}=6$ & 2.960205 & 4.006625 & 14.47315 \\
\hline
\end{tabular}

\subsubsection{Effectiveness of the Proposed e-MNLR Model on the Random-walk Data}

The e-MNLR model presented in Fig. 5 produces equation (5) after applying the calibration process. The equation is then implemented in the $\mathrm{R}$ environment and its accuracy is evaluated in Table 3. As presented in the table, the e-MNLR seems to produce fairly promising results compared to the six counterpart methods.

$$
y_{t}=x_{t}^{0.2271} * x_{d}^{0.0456} * x_{g}^{-0.4465} * x_{p}^{-1.7216} * x_{c}^{3.7746}
$$

Where $y_{t}=$ load at a time (20 minutes), $x_{t}=$ time value whose load is sought, $x_{d}=$ day category number, $x_{g}=$ GDP value of the current year, $x_{p}=$ current population, $x_{c}=$ current number of customers.

Table 3. Performance of the e-MNLR methods as compared to the six counterparts

\begin{tabular}{|c|c|c|c|}
\hline Method & RMSE & MAE & MAPE \\
\hline MNLR & 12.77399 & 9.226775 & 34.34231 \\
\hline Miss-Forest & 3.035463 & 2.732547 & 10.24622 \\
\hline SVM & 5.32299 & 3.15148 & 11.35937 \\
\hline$A N N$ & 6.9456 & 7.466061 & 27.8525 \\
\hline$M I C E$ & 12.09854 & 9.361562 & 33.43858 \\
\hline KNN & 2.960205 & 4.006625 & 14.47315 \\
\hline$e-M N L R$ & 2.109137 & 1.956039 & 7.787976 \\
\hline
\end{tabular}

\subsubsection{The Capability of the Proposed LF-AFDC}

The proposed LF-AFDC framework presented in Fig. 7 is implemented in the $\mathrm{R}$ programming environment. The capability of the developed model to predict a load profile based on the fault and restoration information is then validated. Three testing cases are established in which the fault in the SDEPG is assumed. The information associated with the fault (fault time, fault date, restoration duration) is passed to the LF-AFDC system. In addition to that, other information such as GDP, number of customers, and population are assumed to be acquired in real-time from the designated agencies (NBS and TANESCO).

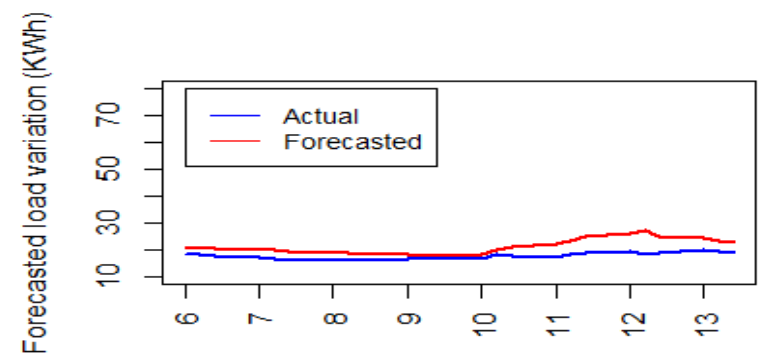

Fig. 10. Test results from LF-AFDC system after predicting load profile based on fault and restoration trigger on (Saturday, 2020-10-03) at 06:00 morning using 8-hours restoration duration 
The first test case is the assumption that the fault has occurred on Saturday, 2020-10-03 at 06:00 AM using an 8hours restoration duration (repair time). From this information, the LF model was able to generate the load profile shown in Fig. 10. As it is seen in the figure, the output is the load profile starting from 6:00 to 13:00 time at 20minute intervals equal to the restoration duration. The GDP, the number of customers, and the population are assumed to be that of the year 2020 .

The second test case is the assumption that the fault has occurred on Sunday (2020-10-04) at 15:00 evening, with restoration duration assumed to be 8 hours. From this information, the LF-AFDC model was able to generate the load profile shown in Fig. 11. As it is seen in the figure, the output is load profile starting from 6:00 to 13:00 time at 20minute intervals equal to restoration duration.

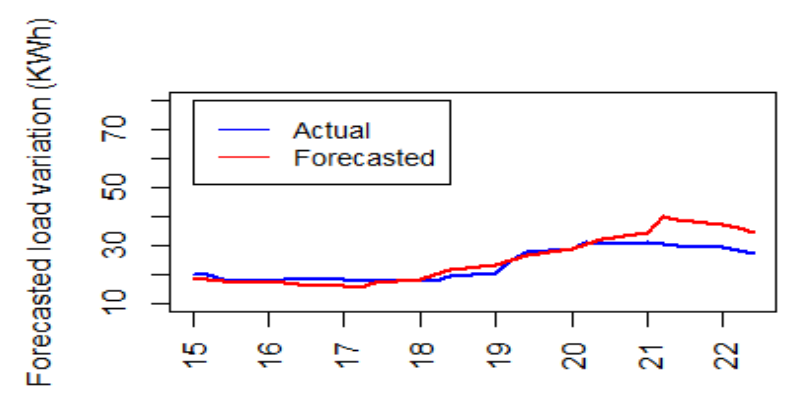

Fig. 11. Test results from LF-AFDC system after predicting load profile based on fault and restoration triggers on 4/10/2020 at 15:00 evening using 8-hours restoration duration

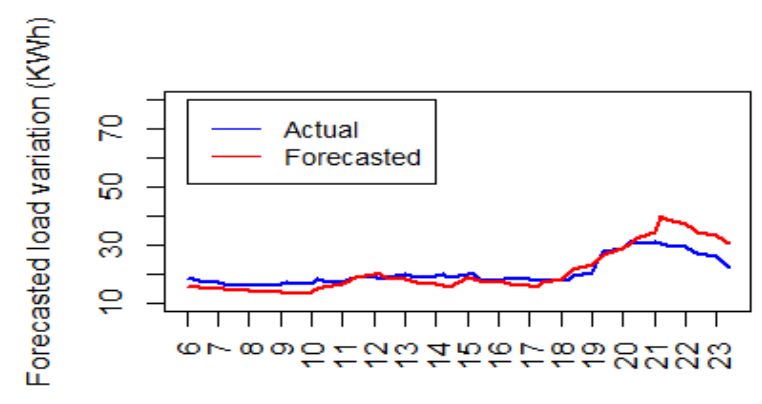

Fig. 12. Test results from the LF system after predicting load profile based on fault and restoration triggers of Friday 2/10/2020 at 06:00 am using an 18-hours restoration duration

The third test case is the assumption that the fault has occurred on Friday 2/10/2020 at 06:00 Am using an 18-hours restoration duration. From this information, the LF-AFDC model was able to generate the load profile shown in Fig. 12. As presented in the figure, the output is the load profile starting from 6:00 to 13:00 time at 20-minute intervals equal to restoration duration.

\subsection{Discussion}

Firstly, this work has revealed the long-term power consumption distribution characteristics in Tanzania, such that future load analysis researchers can rely on. Secondly, this work has also revealed the existence of outliers in the load data such that further load analysis researchers can be aware of when dealing with load analysis works. Thirdly, in this work, the limitation of some widely used prediction methods to the random-walk data has been identified such that load analysts could think of ways to extend the existing methods. Fourthly, load analysis works such as forecasting in the areas whose data is characterized by random walk can adapt the e-MNLR model proposed in this work. Lastly, our LF-AFDC framework has shown great ability to generate load profile from the fault and restoration triggers using both long and short-term factors. This shows that the LF-AFDC model can effectively be applied to predict load demand which can be inferred by other agencies such as DERs and restoration controllers.

\section{Conclusion and Recommendations}

An effective LF-AFDC model that produces load profile from fault (fault time and date) and restoration (expected fault repair time) information in the SDEPG has been proposed in this work. The core forecasting part of the proposed approach has based on the extension of Multivariate Nonlinear Regression to accommodate long-term variables as well as random-walk data. Furthermore, the forecasting part (eMNLR) has been evaluated against two regression-based methods (MICE and MNLR) and four machine learning algorithms (ANN, KNN, SVM, and MissForest). However, despite the capability of the proposed mechanism to produce load profile in the automatic fault clearance using fault and restoration information, the extended Multivariate Nonlinear Regression model has yielded promising results on the random-walk data compared to its counterparts.

This work has focused on developing a load demand forecasting mechanism in LF-AFDC, particularly in residential buildings due to the nature of the study area and available data. In addition to that, the LF-AFDC was expected to acquire the economic (GDP) and demographic indicators (number of customers and population) directly from the NBS and TANESCO agencies. Therefore, future research works may extend the output of this work to accommodate commercial areas as well as to establish direct communication with NBS and TANESCO data centers. Secondly, due to the challenge of data availability, this research is confined to the use of GDP, population, and the number of the customer as major economic factors in the model; for instance, the available electricity price data was just that of few years in such a way that its causality effect on electricity consumption cannot be deduced effectively. 
Therefore, the direction of future work should also focus on extending the model functionality of this research work to include more long-term variables such as electricity price, degree of urbanization, and household economic level.

\section{Acknowledgments}

The authors would like to express their sincere appreciation to any person or institution who at any instance has endeavored to the fulfillment of this research work. Moreover, our heartfelt appreciation goes to the Sida, TANESCO, and UDSM iGrid group for their keen support throughout research implementation.

\section{References}

[1] C. J. Mtokambali and Z. Jun, "Smart Grid Technologies and Future Motivators Influencing Change in the Electricity Sector in Tanzania," Int. J. Sci. Res., vol. 3, no. 2, pp. 39-43, 2014.

[2] G. Chugulu, F. Simba, and S. Lujara, "Proposed Practical Communication Architecture for Automatic Fault Detection and Clearance in Secondary Distribution Power Network," Int. J. Smart Grid ijSmartGrid, vol. 4, no. 4, pp. 165-175, 2020.

[3] D. Mnyanghwalo, S. Kawambwa, R. Mwifunyi, G. M. Gilbert, D. Makota, and N. Mvungi, "Fault Detection and Monitoring in Secondary Electric Distribution Network Based on Distributed Processing," in 2018 20th International Middle East Power Systems Conference, MEPCON 2018 - Proceedings, 2019, no. 1, pp. 84-89, doi: 10.1109/MEPCON.2018.8635141.

[4] R. J. Mwifunyi, M. M. Kissaka, and N. H. Mvungi, "Distributed approach in fault localisation and service restoration: State-of-the-Art and future direction," Cogent Eng., vol. 6, no. 1, pp. 1-20, 2019, doi: 10.1080/23311916.2019.1628424.

[5] M. K. T. A. Kapetanakis, "Short-term Forecast of Automatic Frequency Restoration Reserve from a Renewable Energy Based Virtual Power Plant," Water, vol. 1158, no. 10, pp. 1-20, 2018, doi: 10.3390/w10091158.

[6] V. Donde, Z. Wang, F. Yang, and J. Stoupis, "Shortterm load forecasting based capacity check for automated power restoration of electric distribution networks," 2010 IEEE PES Transm. Distrib. Conf. Expo. Smart Solut. a Chang. World, pp. 1-8, 2010, doi: 10.1109/TDC.2010.5484274.

[7] H. Liu, R. A. Davidson, and T. V. Apanasovich, "Statistical forecasting of electric power restoration times in hurricanes and ice storms," IEEE Trans. Power Syst., vol. 22, no. 4, pp. 2270-2279, 2007, doi: 10.1109/TPWRS.2007.907587.

[8] M. Kleinberg and H. Chiang, "Service Restoration of
Power Distribution Systems Incorporating Load Curtailment," in IEEE International Symposium on Curcuits and Systems, 2009, no. 4, pp. 1709-1712.

[9] A. Zidan, M. Khairalla, A. M. Abdrabou, T. Khalifa, K. Shaban, A. Abdrabou, R. El Shatshat, "Fault Detection , Isolation , and Service Restoration in Distribution Systems : State-of-the-Art and Future Trends," IEEE Trans. Smart Grid, vol. 8, no. 5, pp. 1-16, 2016.

[10] S. Camal, A. Michiorri, G. Kariniotakis, and A. Liebelt, "Short-term forecast of automatic frequency restoration reserve from a renewable energy based virtual power plant," 2017 IEEE PES Innov. Smart Grid Technol. Conf. Eur. ISGT-Europe 2017 - Proc., vol. 2018-Janua, pp. $\quad 1-6, \quad 2017, \quad$ doi: 10.1109/ISGTEurope.2017.8260311.

[11] R. J. Mwifunyi, M. M. Kissaka, and N. H. Mvungi, "Short-Term Load Forecasting for Improved Service Restoration in Electrical Power Systems: A Case of Tanzania," 2020.

[12] E. Kabir, S. D. Guikema, and S. M. Quiring, "Predicting Thunderstorm-Induced Power Outages to Support Utility Restoration," IEEE Trans. Power Syst., vol. 34, no. 6, pp. 4370-4381, 2019, doi: 10.1109/TPWRS.2019.2914214.

[13] G. M. Gilbert and N. Shililiandumi, "Evolutionary Approaches to Fog Node Placement in LV Distribution Networks," Int. J. Smart Grid, vol. 5, no. 1, pp. 23-36, 2021.

[14] M. Rejc, A. Einfalt, and T. Gawron-Deutsch, "Shortterm aggregated load and distributed generation forecast using fuzzy grouping approach," Proc. - 2015 Int. Symp. Smart Electr. Distrib. Syst. Technol. EDST 2015, pp. 212-217, 2015, doi: 10.1109/SEDST.2015.7315209.

[15] M. A. Hammad, B. Jereb, B. Rosi, and D. Dragan, "Methods and Models for Electric Load Forecasting : A Comprehensive Review," Logist. Sustain. Transp., vol. 11, no. 1, pp. 51-76, 2020, doi: 10.2478/jlst-2020-0004.

[16] B. Yildiz, J. I. Bilbao, and A. B. Sproul, "A review and analysis of regression and machine learning models on commercial building electricity load forecasting," Renew. Sustain. Energy Rev., vol. 73, no. December 2016, pp. 1104-1122, 2017, doi: 10.1016/j.rser.2017.02.023.

[17] G. Özbayočlu and M. Evren Özbayočlu, “A new approach for the prediction of ash fusion temperatures: A case study using Turkish lignites," Fuel, vol. 85, no. 4, pp. 545-552, 2006, doi: 10.1016/j.fuel.2004.12.020.

[18] K. Kavaklioglu, "Modeling and prediction of Turkey's electricity consumption using Support Vector Regression," Appl. Energy, vol. 88, no. 1, pp. 368-375, 2011, doi: 10.1016/j.apenergy.2010.07.021. 
[19] S. C. Bhattacharyya and G. R. Timilsina, "Modelling energy demand of developing countries: Are the specific features adequately captured?," Energy Policy, vol. 38, no. 4, pp. 1979-1990, 2010, doi: 10.1016/j.enpol.2009.11.079.

[20] J. Steinbuks, "Assessing the Accuracy of Electricity Demand Forecasts in Developing Countries," Assess. Accuracy Electr. Demand Forecast. Dev. Ctries., 2017, doi: 10.1596/1813-9450-7974.

[21] M. U. Fahad and N. Arbab, "Factor Affecting Short Term Load Forecasting," J. Clean Energy Technol., vol. 2, no. 4, pp. 305-309, 2014, doi: 10.7763/jocet.2014.v2.145.

[22] N. J. Williams, P. Jaramillo, B. Cornell, I. LyonsGalante, and E. Wynn, "Load characteristics of East African microgrids," Proc. - 2017 IEEE PES-IAS PowerAfrica Conf. Harnessing Energy, Inf. Commun. Technol. Afford. Electrif. Africa, PowerAfrica 2017, pp. 236-241, 2017, doi: 10.1109/PowerAfrica.2017.7991230.

[23] G. A. Mabea, "Modelling Residential Electricity Demand for Kenya," J. Econ. Sustain. Dev., vol. 5, no. 4, pp. 145-153, 2014.

[24] R. I. Thamae, L. Z. Thamae, and T. M. Thamae,
"Dynamics of Electricity Demand in Lesotho: A Kalman Filter Approach," Stud. Bus. Econ., vol. 10, no. 1, pp. 130-139, 2015, doi: 10.1515/sbe-2015-0012.

[25] Y. Keho, "What drives energy consumption in developing countries? The experience of selected African countries," Energy Policy, vol. 91, no. December 2015, pp. 233-246, 2016, doi: 10.1016/j.enpol.2016.01.010.

[26] J. Mawejje and D. N. Mawejje, "Electricity consumption and sectoral output in Uganda: an empirical investigation," J. Econ. Struct., vol. 5, no. 1, 2016, doi: 10.1186/s40008-016-0053-8.

[27] H. Bakiri, H. Maziku, N. Mvungi, N. Hamisi, and M. Libe, "Towards the Establishment of Robust Load Forecasting Mechanism in Tanzania Grid : Effect of Air Temperature and Daytime on Electricity Consumption in Residential Buildings," Int. J. Smart Grid, vol. 5, no. 1, pp. 24-36, 2021.

[28] R. Kumari, Sheetanshu, M. K. Singh, R. Jha, and N. K. Singh, "Anomaly detection in network traffic using Kmean clustering," 2016 3rd Int. Conf. Recent Adv. Inf. Technol. RAIT 2016, pp. 387-393, 2016, doi: 10.1109/RAIT.2016.7507933. 